\title{
Building a Successful, Socially-Distanced Family Medicine Clerkship in the COVID Crisis
}

John R. Hayes, DO | Bryan Johnston, MD | Rebecca Lundh, MD

PRiMER. 2020;4:34.

Published: 11/4/2020 | DOI: 10.22454/PRiMER.2020.755864

\section{Abstract}

Introduction: In the wake of the COVID-19 pandemic, many family medicine clerkships across the United States are scrambling to provide meaningful medical education in a new clinical environment. Most medical schools have paused or severely limited the direct patient care that has been the core educational requirement of the clinical clerkships. The aim of this study was to develop and evaluate a curricular model that meets the family medicine clerkship learning objectives via telemedicine clinical instruction.

Methods: We created a clerkship where all clinical encounters took place by phone or video visits. Students were required to have telemedicine encounters with at least 20 patients over a 4-week clerkship. We also created a telemedicine illness script-writing assignment to prepare students and faculty for these encounters. We evaluated our curriculum by surveying participating students and faculty members.

Results: Student surveys revealed that all learning objectives were met during the telemedicine clerkship experience. Students felt they made a difference during the COVID-19 pandemic. Faculty felt that the medical students were helpful and that they were able to provide meaningful clinical instruction.

Conclusion: Our study reveals that a family medicine clerkship can be successfully delivered using telemedicine and remote learning techniques. Senior medical students are a valuable resource and can have a meaningful clinical impact while learning more about family medicine. If social distancing precautions continue, this model can be used by other clerkships to continue medical education and provide medical care.

\section{Introduction}

On March 17, 2020, the Association of American Medical Colleges released guidance that strongly suggested that medical students not be involved in any direct patient care activities, ${ }^{1}$ but medical education continued! Pausing clinical rotations and then having students make up at a later date would have caused an insurmountable backlog of learners for our already-stretched preceptor pool. The crisis inspired rapid innovation and our faculty quickly decided to create an entirely telemedicine-based family medicine clerkship: $100 \%$ of patient care experiences would take place via telephone and video encounters with patients. 


\section{Methods}

For our clerkship's clinical experience, we required students to log at least 20 telemedicine patient encounters over 4 weeks. Encounters were staffed with faculty physicians either asynchronously for low-risk situations or immediately when high-risk medical conditions were discovered. We built flexibility into how exactly each clinical preceptor supplied these 20 encounters. Some faculty members gave students lists of high-risk patients to cold call for COVID-19 education and chronic disease check-ins. Other faculty members chose to directly observe medical students, participating in 3-way phone encounters where students conducted the telemedicine encounters with the faculty for patients scheduled for specific clinic sessions. The clerkship provided additional simulated clinical experiences by requiring our students to complete several Aquifer ${ }^{2}$ cases.

To prepare our clerkship students for telemedicine, we created a "telemedicine illness script writing assignment," due at the end of the first week of the rotation (see supplemental materials for script-writing assignment document). The clerkship provided a template that included an introduction, check-in, and review of symptoms, a placeholder for a 2-minute script on COVID-19, a medication reconciliation, and a placeholder for a 2-minute script on a common chronic disease process. Before writing their scripts, students were instructed to complete a free Centers for Disease Control and Prevention (CDC) module on health literacy for public health professionals, ${ }^{3}$ complete an interactive online COVID case series, ${ }^{4}$ listen to two podcasts that provided up-to-date background information on the COVID-19 infection and response, 5,6 browse the discussion topics on the CDC COVID-19 frequently-asked questions page, ${ }^{7}$ and review a brief telemedicine guide from the Academy of Communication in Healthcare. ${ }^{8}$ These materials provided up-to-date information about COVID-19 in April 2020, but will likely require updating in future versions of this curriculum. After writing their scripts, students were encouraged to review and revise them with their clinical preceptor. After revision, all telemedicine scripts were deidentified and uploaded into a shared drive so they could be used as templates for conversations for all clerkship students. Every student in the clerkship wrote a script for a different chronic disease process, so that we quickly generated a shared library of communication guides for these telemedicine encounters. After completing this preparatory assignment, students were deemed ready to engage with real patients.

We evaluated this remote clerkship curriculum using anonymous surveys via Qualtrics software at the conclusion of the 4-week rotation. Since these surveys fell under the curriculum evaluation/quality improvement umbrella, Medical College of Wisconsin Institutional Review Board members exempted this study from its review process. All faculty members and medical students were also invited to complete short evaluation surveys of their experience with the new remote clerkship (see supplemental materials for student and faculty surveys).

\section{Results}

Fifteen of 17 students and nine preceptors completed the postrotation evaluation. Students conducted an average of 32 clinical patient encounters; this was slightly above the 24-25 encounters that students and preceptors felt should be required of this rotation. Table 1 compares student and preceptor responses to clerkship impact questions. Key findings include that students improved workflow, were capable of assessing for and providing guidance regarding COVID-19, and that many recommended continuing to use telemedicine as part of future in-person clerkships. Nearly all respondents (100\% of preceptors, $93.3 \%$ of students) agreed that this clerkship format enhanced clinical skill development/allowed for meaningful clinical teaching. Table 2 displays student self-evaluation of accomplishment of clerkship learning objectives. Each was evaluated on a 5 -point Likert scale ( $1=$ strongly disagree; $5=$ strongly agree), with mean values of $4.38-4.87$ and standard 
deviation of .40 to 0.78 . This is notable given these learning objectives were unchanged from traditional inperson clerkship. Table 3 compiles themes from student and preceptor responses to free-response prompts, in which five common themes were identified: the students were useful, students and patients benefitted, students developed clinical skills, preceptors identified teaching advantages, and organizational/format challenges of the rotation.

\section{Discussion}

Clinical faculty and medical students in the Medical College of Wisconsin's Family Medicine Clerkship quickly adapted to continue medical education, provide public health information, and care for at-risk patients, all while adhering to the COVID-19 social distancing guidelines. Our entirely telemedicine-based, socially-distant clerkship successfully engaged students in meaningful patient care and met all of our established pre-COVID learning objectives. Our survey of clinical faculty also indicated they were able to continue with excellent clinical instruction while not being overburdened with training responsibilities.

Our clerkship decided that 20 telemedicine encounters, combined with 25 assigned Aquifer cases would provide an adequate learning experience for our leaners. Twenty telemedicine visits is notably less than the 70 physical patient encounters required prior to COVID, so it is somewhat surprising that our survey data were overwhelmingly positive. It is likely that an overall lower volume of clinical cases in our faculty practices allowed for more robust patient care discussions than typically happens in a busy family medicine clinic. Faculty and students also reported there were more opportunities for direct observation and feedback of student-patient communications.

We were fortunate to recruit faculty with extensive student teaching experience to precept clerkship students virtually; all attendings who were invited agreed to participate. Typically, a small proportion of clerkship students are assigned to work with community preceptors, but they were not approached to precept during the initial transformation to telemedicine and virtual teaching at the onset of the pandemic.

Our study has limitations. We present here the evaluation data from only one block rotation of our family medicine clerkship. This was compiled and published rapidly so that other clerkships could learn from our model. We also only had an $88 \%$ response rate on our anonymous student surveys and a $70 \%$ response rate on our faculty surveys. Implementation and further study of this curriculum by other institutions could provide additional validity to our telemedicine curricular model. The authors look forward to collaborating with others as we work together to continue medical education during the COVID-19 pandemic.

\section{Tables and Figures}


Table 1: Comparing Student and Preceptor Quantitative Feedback, by Themes

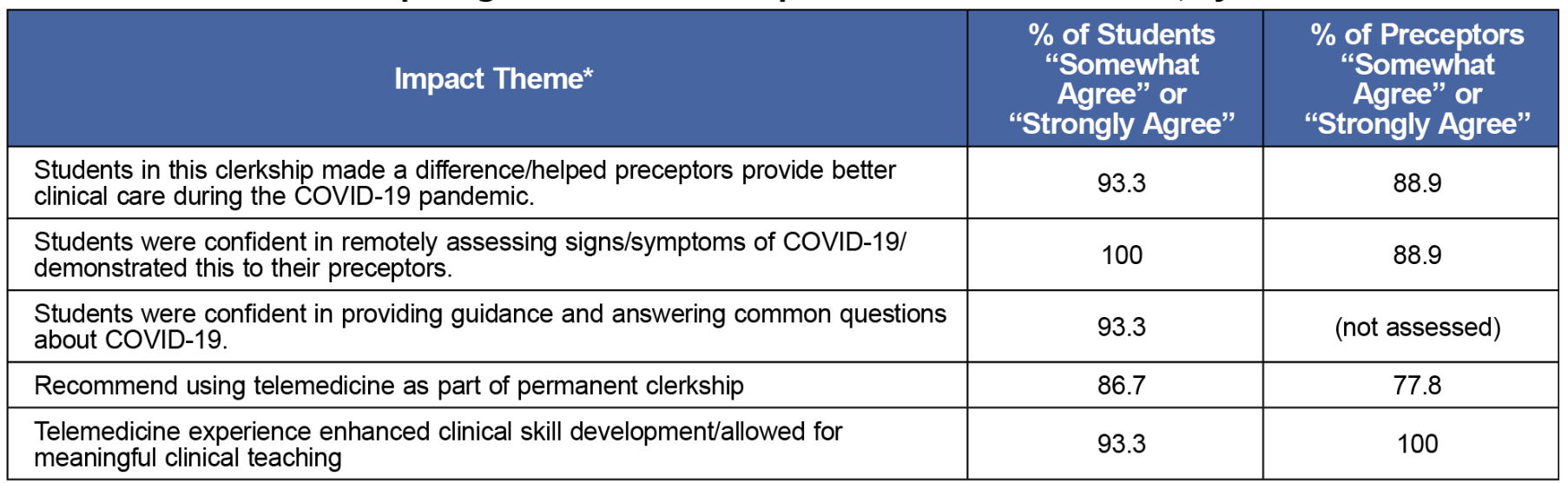

*Assessed on a 5-point likert scale: strongly agree, somewhat agree, neither agree nor disagree, somewhat disagree, strongly disagree.

Table 2: Student Self-assessed Achievement of Clerkship Learning Objectives

\begin{tabular}{|l|c|c|}
\hline \multicolumn{1}{|c|}{$\begin{array}{c}\text { The Family Medicine Clerkship Experience Met } \\
\text { the Following Learning Objectives. I ... }\end{array}$} & Mean (scale 1-5) & SD \\
\hline $\begin{array}{l}\text { Gathered information, formulated differential diagnoses, and proposed plans for initial } \\
\text { evaluation and management of patients with common presentations. }\end{array}$ & 4.73 & 0.44 \\
\hline Managed follow-up visits with patients having one or more common chronic diseases. & 4.87 & 0.50 \\
\hline $\begin{array}{l}\text { Demonstrated competency in advanced elicitation of history, communication, and clinical } \\
\text { reasoning skills. }\end{array}$ & 4.80 & 0.40 \\
\hline Integrated socioeconomic, cultural, and environmental factors into patient care. & 4.73 & 0.57 \\
\hline $\begin{array}{l}\text { Recognized symptoms, signs, and differential diagnoses of common acute and chronic } \\
\text { illnesses as they present in primary care. }\end{array}$ & 4.73 & 0.44 \\
\hline $\begin{array}{l}\text { Developed evidence-based diagnostic and management plans for common acute and } \\
\text { chronic diseases. }\end{array}$ & 4.60 & 0.49 \\
\hline $\begin{array}{l}\text { Integrated population factors of heritage, environment and disease prevalence into patient } \\
\text { care. }\end{array}$ & 15 \\
\hline
\end{tabular}

Scale: 1 (strongly disagree) -5 (strongly agree) 
Table 3: Summary of Student Feedback (In Bold) and Preceptor Feedback (In Italics), Grouped by Themes

\begin{tabular}{|c|c|}
\hline Feedback Themes & Theme Details: Italic: Student Feedback; Bold: Preceptor Feedback \\
\hline Students were useful & $\begin{array}{l}\text { - Felt included in healthcare system efforts to address COVID-19; not the case in other remote } \\
\text { clerkships; "felt so useless at home (before the clerkship)" } \\
\text { - Autonomy appreciated; "made me feel more like a resident than any other rotation" } \\
\text { - Able to experience continuity of care with some patients; "made me feel like I was really integral to } \\
\text { their care" } \\
\text { - Felt the education they provided patients was needed/appreciated } \\
\text { - Students were helpful when preceptors were redeployed; "... called my most at-risk patients } \\
\text { and made a difference in their care"" } \\
\text { - Student history taking and general patient care helped preceptors } \\
\text { - Over } 100 \text { medications orders for chronic conditions were filled at the student-run free clinic } \\
\text { dispensary and provided to patients during the month as a result of these telehealth visits; } \\
\text { additional prescriptions were filled at pharmacies. }\end{array}$ \\
\hline $\begin{array}{l}\text { Students and patients } \\
\text { benefitted from their } \\
\text { interactions }\end{array}$ & $\begin{array}{l}\text { - Enjoyed engaging patients in this way; "Really nice to meet people where they were at" } \\
\text { - Felt patients enjoyed the interactions," "had a lot of... really wonderful patient conversations" } \\
\text { - Felt patients appreciated COVID-19 questions answered from a trusted source } \\
\text { - They were very enthusiastic, which patients appreciated } \\
\text { - Offered students a way to engage with patients } \\
\text { - Patients responded well to telehealth visits }\end{array}$ \\
\hline $\begin{array}{l}\text { Benefits to new teaching } \\
\text { format }\end{array}$ & $\begin{array}{l}\text { - Students had more continuity of care with patients; had more time-flexible patient encounters } \\
\text { - Students learned population health concepts } \\
\text { - Usually more time to teach between patients }\end{array}$ \\
\hline $\begin{array}{l}\text { Organizational/format } \\
\text { challenges }\end{array}$ & $\begin{array}{l}\text { - Not enough clinical encounters of different kinds: acute care, chronic disease management, pediatrics } \\
\text { - Not enough scheduled encounters/too much pop health exposure } \\
\text { - Inconsistent organization/lead time for changes/assignments } \\
\text { - Not enough formative feedback or attending teaching } \\
\text { - Scheduling/timing were challenges } \\
\text { - Difficult to assess students; limited observation of their skills } \\
\text { - Concerns that breadth of family medicine not translated }\end{array}$ \\
\hline
\end{tabular}

\section{Acknowledgments}

The authors thank the following clinical faculty members for their dedication to family medicine medical student education: Leslie Ruffalo, Courtney Barry, Craig Young, Joseph Gravel, Lauren Bauer, Brooke Passolt, Sabina Diehr, Karen Hulbert, David Johnson, Laura Brusky, Rebecca Bernstein, Seth Bodden, Robin Helm, Veneshia McKinney, Kwanza Devlin, and Daniel Duffy. They also thank their clerkship staff Stephanie Shaw and Alanda Malveaux for their hard work making this project a reality.

\section{Corresponding Author}

John R. Hayes, DO

Medical College of Wisconsin, Department of Community and Family Medicine, 8701 W. Wauwatosa, WI 53211 jrhayes@mcw.edu

\section{Author Affiliations}

John R. Hayes, DO - Medical College of Wisconsin, Department of Community and Family Medicine Bryan Johnston, MD - Medical College of Wisconsin, Department of Community and Family Medicine Rebecca Lundh, MD - Medical College of Wisconsin, Department of Community and Family Medicine

\section{References}

1. Whelan A, Prescott J, Young G, Catanese V, McKinney R. Guidance on Medical Students' Participation in 
Direct Patient Contact Activities. https://www.aamc.org/system/files/2020-04/meded-April-14-Guidanceon-Medical-Students-Participation-in-Direct-Patient-Contact-Activities.pdf. Washington, DC: Association of American Medical Colleges; April 14, 2020. Updated August 14, 2020. Accessed November 3, 2020.

2. Family Medicine: A case-based virtual course. https://aquifer.org/courses/aquifer-family-medicine/. Accessed November 3, 2020.

3. CDC Train. Health Literacy for Public Health Professionals. https://www.train.org/cdctrain/course /1078759. Accessed November 3, 2020.

4. Aultman A, Levy J, Neipris L. Case Network: COVID-19 Patient Simulation. Retrieved from http://casenetwork.com/webcontent/covid/\#/. Accessed November 3, 2020.

5. Domino F. primed. 03/21/20. Frankly Speaking Update: Common Questions about Coronavirus [audio podcast]. https://www.pri-med.com/online-education/podcast/frankly-speaking-cme-coronavirus. March 21, 2020. Accessed November 3, 2020.

6. Ganatra R, Watto M, Williams P, Sax P. Curbsiders. 03/22/20. \#200 COVID-19 Update with Paul Sax MD: Thoughts on Transmission, Quarantine, PPE, Diagnosis and Management [audio podcast]. https://thecurbsiders.com/podcast/200. March 22, 2020. Accessed November 3, 2020.

7. Centers for Disease control and Prevention. Coronavirus Disease 2019 - Frequently Asked Questions. https://www.cdc.gov/coronavirus/2019-ncov/faq.html. Updated October 29, 2020. Accessed November 3, 2020.

8. Academy of Communication in Healthcare. COVID-19 Telehealth Communication Guide. 03/2020. https://www.achonline.org/COVID-19/Telemedicine. March, 2020. Accessed November 3, 2020.

9. Doximity Dialer Smartphone Application. https://www.doximity.com/clinicians/download/dialer. Accessed November 3, 2020.

Copyright $(\mathcal{2} 2020$ by the Society of Teachers of Family Medicine 